\title{
Abbreviated Protocols versus Multiparametric MRI for Assessment of Extraprostatic Extension in Prostatic Carcinoma: A Multireader Study
}

\author{
ARNALDO STANZIONE ${ }^{*}$, ANDREA PONSIGLIONE ${ }^{1 *}$, RENATO CUOCOLO $^{1}$, \\ SIRIO COCOZZA ${ }^{1}$, STEFANO GIUSTO PICCHI ${ }^{1}$, SALVATORE STILO ${ }^{1}$, FRANCESCO PERSICO $^{2}$, \\ MASSIMILIANO CRETA ${ }^{2}$, NICOLA LONGO ${ }^{2}$ and MASSIMO IMBRIACO ${ }^{1}$ \\ ${ }^{1}$ Department of Advanced Biomedical Sciences, University of Naples "Federico II", Naples, Italy; \\ ${ }^{2}$ Department of Neurosciences, Reproductive Sciences and Odontostomatology, \\ University of Naples "Federico II", Naples, Italy
}

\begin{abstract}
Background/Aim: Prostate multiparametric magnetic resonance imaging ( $m p M R I)$ is the reference imaging modality for extraprostatic extension of disease (EPE) assessment. We aimed to compare the diagnostic accuracy of different abbreviated MRI protocols to the standard prostate mpMRI in the identification of EPE of PCa. Patients and Methods: Fifty patients were retrospectively enrolled. Dualpulse (dpMRI) and biparametric (bpMRI) abbreviated protocols were obtained from mpMRI. The performance of two experienced radiologists and two radiology residents was correlated with a reference standard and compared. Inter and intra-reader agreements were evaluated. Results: All protocols were strongly correlated to the reference standard $(p \leq 0.001)$. A significant difference was found between dpMRI and mpMRI $(p=0.009)$, no differences emerged between bpMRI and mpMRI $(p=0.27)$. All readers showed moderate agreement ( $k=0.47$, $\kappa=0.50$ and $k=0.53$ for dpMRI, bpMRI and mpMRI, respectively). Intra-reader agreement was good (all $k$ values 20.70). Conclusion: Only bpMRI showed similar diagnostic performance to mpMRI, thus appearing as a feasible alternative to the standard protocol for EPE detection.
\end{abstract}

Local staging is a crucial phase in the management of patients affected by prostate cancer (PCa). Indeed, an

\footnotetext{
*Both authors contributed equally to this article.

Correspondence to: Renato Cuocolo, MD, Department of Advanced Biomedical Sciences, University of Naples "Federico II", Via S. Pansini, 5, 80131, Naples, Italy. Tel: +39 0817463561, Fax: +39 0815457081,e-mail: renato.cuocolo@unina.it
}

Key Words: Prostate cancer, multiparametric MRI, extraprostatic extension. accurate assessment of extraprostatic extension (EPE) of the disease could modify the decision of an appropriate treatment strategy and therapeutic planning (1).

In this context, magnetic resonance imaging (MRI) is regarded as one of the best imaging modalities to achieve a correct and reliable pre-treatment local staging of PCa (2-4). In particular, multiparametric MRI (mpMRI), which is composed of three planes, T2-weighted (T2-w), axial diffusion weighted imaging (DWI) and axial dynamic contrast enhanced (DCE) sequences, is to date acknowledged as the standardized and recommended imaging protocol for the detection and local staging of PCa (5). Nevertheless, alternative short imaging protocols have been recently proposed as valuable alternatives to the standard mpMRI (6-9). Aims of these abbreviated protocols were to reduce acquisition time, costs and patients' discomfort, mainly diverging from mpMRI due to the removal of the DCE sequence. This protocol, referred as biparametric MRI (bpMRI), has been proven to have a similar diagnostic accuracy in the detection of PCa $(6,7,9,10)$. Along with bpMRI, even shorter protocols have been suggested, occasionally referred as dual-pulse MRI (dpMRI), which rely on the sole evaluation of axial T2-w and DWI sequences, further removing the assessment of coronal and sagittal T2-w images (11-13).

While both dpMRI and bpMRI have demonstrated, compared to mpMRI, a similar good diagnostic accuracy in the detection of $\mathrm{PCa}$, no evidence is available regarding their possible different performances in local staging. Therefore, the aim of this study was to evaluate the diagnostic performance of different abbreviated MRI protocols compared to the standard prostate mpMRI in the identification of EPE of $\mathrm{PCa}$, and also assess the "real world" reproducibility of these different MRI protocols evaluating the diagnostic performances obtained by four independent readers with different levels of expertise. 


\section{Patients and Methods}

This observational, retrospective study was approved by the Institutional Review Board (Protocol number: 60/19) and the need for informed consent was waived.

Patient population. We selected 41 consecutive patients diagnosed with PCa by means of biopsy who underwent a standard mpMRI at our Institution to assess local stage prior to radical prostatectomy (RP) between January 2016 and October 2017. To avoid reader bias, we also included a second category of consecutive patients $(n=15)$ with clinical suspicion of $\mathrm{PCa}$ and a negative biopsy result, who underwent a mpMRI exam to guide a re-biopsy that confirmed the absence of PCa. Six subjects were excluded due to incomplete or inadequate (e.g. presence of artefacts) mpMRI, or because RP was performed more than three months after mpMRI, leading a final number of 50 patients. The following demographic and clinical data were recorded: patients' age, Prostate Specific Antigen (PSA) levels, pathological data including Gleason Score and local stage according to the TNM classification of malignant tumors (Table I).

MRI data acquisition. A complete list of the different MRI acquisition parameters and protocols is shown in Table II. All examinations were performed on a 3 Tesla scanner without endorectal coil (Magnetom Trio, Siemens Healthcare, Erlangen, Germany).

Briefly, from the standard mpMRI images acquired according to the PI-RADS v2 recommendations (5), two different unenhanced MRI protocols were extracted, dpMRI composed of axial T2-w and a DWI (with the corresponding ADC maps) sequences, and bpMRI protocol included three-planes T2-w sequences along with an axial DWI. The different structure of MRI protocols is highlighted in Figure 1.

Image analysis. Images were independently reviewed by two experienced radiologists (ERs) highly trained in MR prostatic imaging (both with more than 5 years of experience) and two radiology residents (RRs) (both with less than 3 years of experience in prostate imaging).

Readers were asked to independently review images from each protocol of every patient included in the study, blinded to clinical information, and to assess first if an index lesion was present (i.e. PI-RADSv2 score $\geq 3$ ) and, in case of positive results, if it was suggestive for prostate confined disease or EPE. The second assessment was also performed, in accordance to the PI-RADSv2 indications, evaluating the presence of both direct and indirect signs (e.g. length of capsule contact, bulging) to the make the final decision (5). For all evaluations of each reader, reviewing time expressed in seconds (sec) was recorded.

Initially, the four readers evaluated the dpMRI protocol, while bpMRI and mpMRI protocols were reviewed after a 5-weeks washout, after completing each evaluation. To further reduce readers' bias, for each protocol multiple reading sessions were organized, and the images administered to the reader were randomly ordered and grouped.

Furthermore, to evaluate intra-reader agreement, one ER and one $\mathrm{RR}$ re-evaluated all the images from each protocol after an additional 5-week period.

Statistical analysis. Image evaluation times for each protocol, averaged among experience groups, were compared using a twosample $t$-test.
Table I. Patient population demographic and clinical information.

\begin{tabular}{lcc}
\hline Characteristics & $\begin{array}{c}\text { PCa affected } \\
\text { patients }(\mathrm{n}=35)\end{array}$ & $\begin{array}{c}\text { PCa free } \\
\text { patients }(\mathrm{n}=15)\end{array}$ \\
\hline Age (years), mean (SD) & $66.2(5.4)$ & $62.7(8.8)$ \\
PSA level (ng/ml), mean (SD) & $8.1(3.6)$ & $7.3(3.3)$ \\
Gleason score at RP & & \\
$6(3+3), \mathrm{n}(\%)$ & $4(11.5)$ & N/A \\
$7(3+4), \mathrm{n}(\%)$ & $11(31.4)$ & N/A \\
$7(4+3), \mathrm{n}(\%)$ & $11(31.4)$ & N/A \\
$8(4+4), \mathrm{n}(\%)$ & $6(17.1)$ & N/A \\
$\geq 9, \mathrm{n}(\%)$ & $3(8.6)$ & N/A \\
Histopathological local stage & & \\
T2a, n(\%) & $3(8.6)$ & N/A \\
T2b, n(\%) & $1(2.8)$ & N/A \\
T2c, n(\%) & $15(42.9)$ & N/A \\
T3a, n(\%) & $14(40.0)$ & N/A \\
T3b, n(\%) & $2(5.7)$ & N/A \\
\hline
\end{tabular}

N/A: Not applicable; PCa: prostate cancer; PSA: prostate-specific antigen; RP: radical prostatectomy; SD: standard deviation.

Fleiss' Kappa (к) was calculated to evaluate agreement between readers in the assessment of each different protocol, while Cohen's $\mathrm{K}$ was used to evaluate both the inter-reader agreement between ERs and RRs for each protocol, as well as the intra-reader agreement in the two-step evaluation performed for every MRI protocol. All agreements were interpreted, on the base of their $\mathrm{K}$ values, using the following classification: $\leq 0.20$ : poor agreement; $0.20-0.40$ : fair agreement; 0.40-0.60: moderate agreement; 0.60-0.80: good agreement; $\geq 0.80$ : excellent agreement (14).

A Spearman analysis was performed to evaluate the correlation of each reader, when reviewing the different protocols, to the reference standard. Correlation strength was classified as low, moderate, high and excellent using the following correlation coefficient cut-off values respectively: $\leq 0.35$, between 0.36 and 0.67 , between 0.68 and 0.90 , and $\geq 0.91$. Data were tested to verify normal distribution using the Kolmogorov-Smirnov test. Subsequently, correlation coefficients of dpMRI and bpMRI were compared to the ones obtained with the mpMRI protocol using a paired $t$-test.

Measures of diagnostic performances (i.e. sensitivity, specificity, positive and negative predictive values and diagnostic accuracy) in the identification of EPE were calculated for each different reader and every MRI protocol, and these measures were then averaged across experience group readers.

\section{Results}

For all readers, mean average time for reviewing dpMRI, bpMRI and mpMRI was respectively 222, 262 and $290 \mathrm{sec}$; the time for reviewing dpMRI was significantly lower compared to mpMRI $(p \leq 0.001)$, while no difference was found between bpMRI and mpMRI $(p=0.06)$. When evaluating differences between ERs and RRs in the time needed for the evaluation of the three protocols, significant differences emerged for dpMRI (163vs. $281 \mathrm{sec} ; p \leq 0.001)$ 


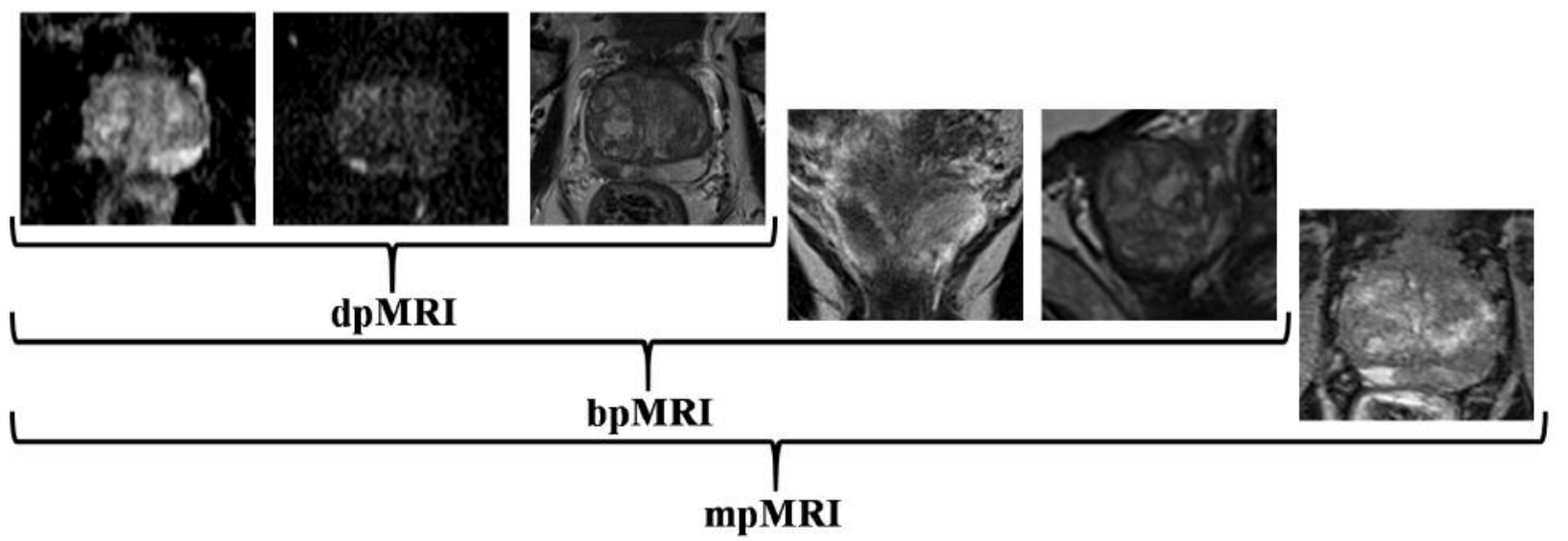

Figure 1. MR images of a 74-year-old patient with a lesion suspicious for prostate cancer located in the right peripheral zone. From left to right, axial ADC map, axial high b-value DWI, axial T2-weighted, coronal T2-weighted, sagittal T2-weighted and axial DCE sequences are shown. The structure of the three different protocols is highlighted by braces. Interestingly, in the coronal T2-weighted sequence (4th image from left) the real maximum diameter of the suspicion lesion is revealed. Radical prostatectomy revealed a 4+4 Gleason score prostate cancer, with absence of extraprostatic extension of disease.

Table II. Technical parameters of each MRI sequence, with + and - signs marking whether the sequence is included or excluded in the protocol, respectively.

\begin{tabular}{|c|c|c|c|c|c|}
\hline & $\begin{array}{l}\text { Axial } \\
\text { DWI }\end{array}$ & $\begin{array}{l}\text { Axial } \\
\text { T2-w }\end{array}$ & $\begin{array}{l}\text { Sagittal } \\
\text { T2-w }\end{array}$ & $\begin{array}{l}\text { Coronal } \\
\text { T2-w }\end{array}$ & DCE \\
\hline dpMRI & + & + & - & - & - \\
\hline bpMRI & + & + & + & + & - \\
\hline mpMRI & + & + & + & + & + \\
\hline TR (ms) & 4900 & 4000 & 4000 & 4000 & 4.38 \\
\hline TE (ms) & 89 & 101 & 101 & 101 & 1.55 \\
\hline Slice thickness $(\mathrm{mm})^{*}$ & 3 & 3 & 3 & 3 & 3 \\
\hline Matrix & $144 \times 144$ & $310 \times 320$ & $310 \times 320$ & $310 \times 320$ & $115 \times 192$ \\
\hline FOV (mm) & $200 \times 200$ & $200 \times 200$ & $200 \times 200$ & $200 \times 200$ & $260 \times 260$ \\
\hline
\end{tabular}

DWI: Diffusion weighted imaging; DCE: dynamic contrast enhanced; T2-w: T2-weighted; dpMRI: dual-pulse MRI; bpMRI: biparametric MRI; mpMRI: multiparametric MRI; TR: repetition time; TE: echo time; FOV: field of view. DWI was performed with b values of 50, 800 and 1,500 $\mathrm{sec} / \mathrm{mm}^{2}$. *no gap between slices.

and bpMRI ( 232 vs. $293 \mathrm{sec} ; p=0.001)$, but not for mpMRI (270 vs. $310 \mathrm{sec} ; p=0.06$ ). Interestingly, ERs showed a greater increase in reading time when comparing both dpMRI to bpMRI and bpMRI to mpMRI (69 and $38 \mathrm{sec}$ ) compared to RRs (12 and $17 \mathrm{sec}$ ).

The four readers showed a moderate agreement among each protocol $(\mathrm{K}=0.47, \mathrm{~K}=0.50$ and $\mathrm{k}=0.53$ for dpMRI, bpMRI and mpMRI, respectively). When agreement within groups was tested considering the experience of the readers, an increase in the agreement was found for the ERs $(\mathrm{k}=0.48$, $\mathrm{k}=0.65$ and $\mathrm{k}=0.73$ for dpMRI, bpMRI and mpMRI, respectively), while RRs showed a more stable, although lower agreement $(\mathrm{k}=0.42, \mathrm{k}=0.42$ and $\mathrm{k}=0.46$ for dpMRI, bpMRI and mpMRI, respectively). Finally, the intra-reader agreement analysis showed high values of concordance (all
$\mathrm{K}$ values $\geq 0.70$ ). In particular, the ER obtained $\mathrm{K}$ values of $0.70,0.78$ and 0.73 while the RR scored $\mathrm{k}$ values of 0.79 , 0.94 and 0.72 for dpMRI, bpMRI and mpMRI, respectively.

Results of the correlation analysis showed that all protocols were strongly correlated to the reference standard (Table III), with a trend towards an increase in correlation from dpMRI to mpMRI. When differences between protocols were tested, a significant difference emerged between dpMRI and mpMRI ( $p=0.009)$, while no differences emerged for the dpMRI versus bpMRI and bpMRI versus mpMRI comparisons ( $p=0.11$ and $p=0.27$, respectively).

Sensitivity, specificity, positive and negative predictive value, along with diagnostic accuracy among groups of experience for each protocol are presented in Table IV. Briefly, all the protocols reached a similar high performance 
Table III. Results of correlation analyses between different MRI protocols and reference standard. All results are with $p<0.001$.

\begin{tabular}{llll}
\hline & dpMRI & bpMRI & mpMRI \\
\hline ER 1 & $\mathrm{r}=0.797$ & $\mathrm{r}=0.854$ & $\mathrm{r}=0.842$ \\
ER 2 & $\mathrm{r}=0.779$ & $\mathrm{r}=0.870$ & $\mathrm{r}=0.883$ \\
RR 1 & $\mathrm{r}=0.512$ & $\mathrm{r}=0.503$ & $\mathrm{r}=0.587$ \\
RR 2 & $\mathrm{r}=0.494$ & $\mathrm{r}=0.543$ & $\mathrm{r}=0.567$ \\
\hline
\end{tabular}

ER: Experienced radiologist; RR: radiology resident; dpMRI: dual-pulse MRI; bpMRI: biparametric MRI; mpMRI: multiparametric MRI.

in terms of diagnostic accuracy, with consistently expected lower performances for the RRs compared to the ERs. Among the three protocols, dpMRI proved to have slightly lower diagnostic accuracy (ERs $=83.0 \%$; RRs $=75.0 \%$ ), while similar values were found for bpMRI (ERs $=86.0 \%$; RRs=75.0\%) and mpMRI (ERs=87.0\%; RRs=77.0\%), with a subtle trend of increase when readers evaluated more complex imaging protocols (ranging from $83 \%$ to $87 \%$ for ERs and from $75 \%$ to $77 \%$ for RRs).

\section{Discussion}

In this study, we investigated the performance of readers with different expertise in the evaluation of three prostate MRI protocols for the identification of EPE, in patients with PCa. Our results indicate that bpMRI is comparable to mpMRI. On the other hand, dpMRI proved to have lower reproducibility and worse correlation with histopathology, suggesting that standard mpMRI could be shortened by eliminating DCE sequences, while the remaining sequences should be acquired.

The mpMRI protocol, including multiplanar T2-w, DWI and DCE sequences, is considered as the current standard for prostate MR imaging (5). Nevertheless, its composition has been debated in recent years, with particular reference to the use of DCE sequences for the detection of PCa (15-17). Indeed, it has been demonstrated that removing it from prostate MRI would significantly improve cost-effectiveness without a significant loss in terms of diagnostic accuracy (18). Moreover, this approach would also reduce the possible risks associated with multiple gadolinium contrast agent administrations (19, 20). In this context, the search of alternative unenhanced, shorter imaging protocols has been the area of interest of different research groups, although being mostly focused on PCa detection $(6-9,11,21,22)$.

In addition, of those that attempted to verify the efficiency of shortened prostate MRI protocols in the evaluation of EPE, to the best of our knowledge, none has made a direct comparison with mpMRI (23-25). Nevertheless, these studies suggested a possible role of bpMRI in this field, as confirmed in this work.
Table IV. Indexes of diagnostic performances for readers averaged by experience among the three different MRI protocols.

\begin{tabular}{lcccccc}
\hline \multirow{2}{*}{} & \multicolumn{2}{c}{ dpMRI } & \multicolumn{2}{c}{ bpMRI } & \multicolumn{2}{c}{ mpMRI } \\
\cline { 2 - 7 } & ERs & \multirow{2}{*}{ RRs } & ERs & \multirow{2}{*}{ RRs } & ERs & \multirow{2}{*}{ RRs } \\
\hline Sensitivity & $87.0 \%$ & $78.7 \%$ & $88.2 \%$ & $78.8 \%$ & $88.9 \%$ & $80.9 \%$ \\
Specificity & $83.3 \%$ & $64.0 \%$ & $86.8 \%$ & $65.0 \%$ & $84.1 \%$ & $66.7 \%$ \\
PPV & $89.7 \%$ & $86.8 \%$ & $92.6 \%$ & $86.8 \%$ & $92.6 \%$ & $86.8 \%$ \\
NPV & $68.8 \%$ & $50.0 \%$ & $71.9 \%$ & $50.0 \%$ & $75.0 \%$ & $56.3 \%$ \\
Diagnostic accuracy & $83.0 \%$ & $75.0 \%$ & $86.0 \%$ & $75.0 \%$ & $87.0 \%$ & $77.0 \%$ \\
\hline
\end{tabular}

ERs: Experienced radiologists; RRs: radiology residents; dpMRI: dualpulse MRI; bpMRI: biparametric MRI; mpMRI: multiparametric MRI; PPV: positive predictive value; NPV: negative predictive value.

The main result of our study is the absence of significant difference in terms of correlation with the reference standard, between mpMRI and bpMRI, as opposed to dpMRI, that showed a significantly lower correlation when compared to mpMRI. This suggests that the identification of EPE might be equally performed either with bpMRI or mpMRI, while dpMRI does not seem appropriate for this task. Therefore, we hypothesize that while DCE removal can be mitigated by the availability of two other axial sequences, providing both functional and anatomical information, the availability of sagittal and coronal T2-w images significantly impacts proper evaluation of EPE in PCa patients. Interestingly, when a direct comparison between dpMRI and bpMRI was performed, no statistically significant differences in terms of correlation with the reference standard were obtained, although we wish to highlight that mean performances were higher for bpMRI compared to dpMRI.

When investigating the role of experience in EPE detection, we found that all correlation coefficients in our study proved to be relatively high (ranging from $r=0.494$ to $r=0.883$ ). Nevertheless, correlation strength was moderate for the RR and high for the ER group. This result, somewhat expected, is in agreement with previous studies suggesting that experience level has an important role in PCa EPE assessment $(25,26)$, a complex task more suitable for readers with adequate expertise in the field. In contrast to these results, recently Jansen $\mathrm{et} \mathrm{al}$. reported the absence of a learning curve for EPE identification on mpMRI (27) probably due to the background of readers involved in the study. Indeed, as stated by the authors, only the performance over time of radiologists with an already strong expertise in prostatic imaging was evaluated.

Furthermore, the overall inter-reader agreement for all protocols in our study was moderate, again in line with previous studies $(24,28)$. On the other hand, when assessing reproducibility across protocols based on experience level, inter-reader agreement was good for bpMRI and mpMRI and moderate for dpMRI in the ER group, while a moderate agreement for all protocols, was obtained by the RR group. 
Diagnostic accuracy was also higher in the ER group compared to RRs across all evaluated protocols, as shown in Table IV. These results further corroborate our hypothesis about the incremental value of expertise in prostatic imaging. Interestingly, the diagnostic performance of mpMRI in our study differs from those found in a recent meta-analysis (29). This could be at least partially explained by the presence of PCa-free subjects in our patient population that might have contributed increasing sensitivity.

It is interesting to note that ERs showed a greater increase in reading time when comparing both dpMRI to bpMRI and bpMRI to mpMRI (69 and $38 \mathrm{sec}$ ) compared to RRs (12 and $17 \mathrm{sec})$. We believe that this could be related to an overreliance of RRs on axial images, albeit the presence of longer dpMRI reading times. This is also reflected in their similar performance across all prostate imaging protocols. The performance gap that is due to experience level may be filled by the development of computer aided diagnosis software dedicated to EPE detection. Indeed, in the recent literature, radiomics and machine learning have shown promising results in this field and their added value for lesser-experienced readers should be investigated in future studies (30-32).

This study suffers from several limitations that should be acknowledged. The number of subjects included is relatively low for a multi-reader study and a greater sample size would have allowed to perform additional evaluations such as differentiating seminal vesicle, neurovascular-bundle and adjacent organs invasion; furthermore, it was not possible to separate patients in subgroups according to risk stratification (1). Conversely, a low number of patients can be partly justified by the mandatory need for radical prostatectomy as our reference standard. Although several strategies have been embraced to reduce reader bias, it is not possible to completely exclude its presence due to the relatively low number of cases and repeated reading sessions.

In conclusion, evidence collected in this study suggests that bpMRI might be adopted as a valid alternative to mpMRI for the identification of EPE; on the other hand, dpMRI does not appear as accurate for this purpose. Our results also indicate that, regardless of MRI protocol characteristics, radiologic experience significantly influences the performance of readers.

\section{Conflicts of Interest}

The Authors have no conflicts of interest to declare regarding this study.

\section{Authors' Contributions}

Guarantor of integrity of the entire study: Massimo Imbriaco; Study concepts and design: Arnaldo Stanzione; Andrea Ponsiglione; Literature research: Arnaldo Stanzione; Andrea Ponsiglione; Renato Cuocolo; Clinical studies: Stefano Giusto Picchi; Salvatore Stilo; Francesco Persico; Massimiliano Creta; Nicola Longo; Data analysis:
Arnaldo Stanzione; Andrea Ponsiglione; Renato Cuocolo; Sirio Cocozza; Statistical analysis: Sirio Cocozza; Manuscript preparation: Arnaldo Stanzione; Andrea Ponsiglione; Renato Cuocolo; Sirio Cocozza; Massimo Imbriaco; Manuscript editing: Nicola Longo; Massimo Imbriaco.

\section{References}

1 Mottet N, Bellmunt J, Bolla M, Briers E, Cumberbatch MG, De Santis M, Fossati N, Gross T, Henry AM, Joniau S, Lam TB, Mason MD, Matveev VB, Moldovan PC, van den Bergh RCN, Van den Broeck T, van der Poel HG, van der Kwast TH, Rouvière O, Schoots IG, Wiegel T and Cornford P: EAU-ESTRO-SIOG Guidelines on Prostate Cancer. Part 1: Screening, diagnosis, and local treatment with curative intent. Eur Urol 71: 618-629, 2017. PMID: 24207135. DOI: 10.1016/j.eururo.2016. 08.003

2 Duvnjak P, Schulman AA, Holtz JN, Huang J, Polascik TJ and Gupta RT: Multiparametric prostate MR imaging: Impact on clinical staging and decision making. Urol Clin North Am 45: 455466, 2018. PMID: 29420979. DOI: 10.1016/j.ucl.2018. 03.010

3 Barret E, Turkbey B, Puech P, Durand M, Panebianco V, Fütterer JJ, Renard-Penna R and Rouvière O: Update on the ICUD-SIU consultation on multi-parametric magnetic resonance imaging in localised prostate cancer. World J Urol 37: 429-436, 2019. PMID: 30003373. DOI: $10.1007 / \mathrm{s} 00345-018-2395-3$

4 McEvoy SH, Raeside MC, Chaim J, Ehdaie B and Akin O: Preoperative prostate MRI: A road map for surgery. Am J Roentgenol 211: 383-391, 2018. PMID: 29894214. DOI: 10.2214/AJR.17.18757

5 Barentsz JO, Weinreb JC, Verma S, Thoeny HC, Tempany CM, Shtern F, Padhani AR, Margolis D, Macura KJ, Haider MA, Cornud F and Choyke PL: Synopsis of the PI-RADS v2 Guidelines for multiparametric prostate magnetic resonance imaging and recommendations for use. Eur Urol 69: 41-49, 2016. PMID: 26361169. DOI: 10.1016/j.eururo.2015.08.038

6 Obmann VC, Pahwa S, Tabayayong W, Jiang Y, O'Connor G, Dastmalchian S, Lu J, Shah S, Herrmann KA, Paspulati R, MacLennan G, Ponsky L, Abouassaly R and Gulani V: Diagnostic accuracy of a rapid biparametric MRI protocol for detection of histologically proven prostate cancer. Urology 122: 133-138, 2018. PMID: 30201301. DOI: 10.1016/j.urology. 2018.08.032

7 De Visschere P, Lumen N, Ost P, Decaestecker K, Pattyn E and Villeirs G: Dynamic contrast-enhanced imaging has limited added value over T2-weighted imaging and diffusion-weighted imaging when using PI-RADSv2 for diagnosis of clinically significant prostate cancer in patients with elevated PSA. Clin Radiol 72: 23-32, 2017. PMID: 27726850. DOI: 10.1016/ j.crad.2016.09.011

8 Di Campli E, Delli Pizzi A, Seccia B, Cianci R, D'Annibale M, Colasante A, Cinalli S, Castellan P, Navarra R, Iantorno R, Gabrielli D, Buffone A, Caulo M and Basilico R: Diagnostic accuracy of biparametric vs multiparametric MRI in clinically significant prostate cancer: Comparison between readers with different experience. Eur J Radiol 101: 17-23, 2018. PMID: 29571792. DOI: $10.1016 / j$.ejrad.2018.01.028

9 Stanzione A, Imbriaco M, Cocozza S, Fusco F, Rusconi G, Nappi C, Mirone V, Mangiapia F, Brunetti A, Ragozzino A and Longo $\mathrm{N}$ : Biparametric 3T Magentic Resonance Imaging for prostatic cancer detection in a biopsy-naïve patient population: a further improvement of PI-RADS v2? Eur J Radiol 85: 2269-2274, 2016. DOI: $10.1016 /$ j.ejrad.2016.10.009 
10 Cuocolo R, Stanzione A, Rusconi G, Petretta M, Ponsiglione A, Fusco F, Longo N, Persico F, Cocozza S, Brunetti A and Imbriaco M: PSA-density does not improve bi-parametric prostate MR detection of prostate cancer in a biopsy naïve patient population. Eur J Radiol 104: 64-70, 2018. PMID: 29857868. DOI: 10.1016/j.ejrad.2018.05.004

11 Barth BK, De Visschere PJL, Cornelius A, Nicolau C, Vargas HA, Eberli D and Donati OF: Detection of clinically significant prostate cancer: short dual-pulse sequence versus standard multiparametric MR imaging - A multireader study. Radiology 284: 725-736, 2017. PMID: 28346073. DOI: 10.1148/radiol. 2017162020

12 Kuhl CK, Bruhn R, Krämer N, Nebelung S, Heidenreich A and Schrading S: Abbreviated biparametric prostate MR imaging in men with elevated prostate-specific antigen. Radiology 285: 493505, 2017. PMID: 28727544. DOI: 10.1148/radiol.2017 170129

13 Gaur S, Harmon S, Gupta RT, Margolis DJ, Lay N, Mehralivand S, Merino MJ, Wood BJ, Pinto PA, Shih JH, Choyke PL and Turkbey B: A multireader exploratory evaluation of individual pulse sequence cancer detection on prostate multiparametric magnetic resonance imaging (MRI). Acad Radiol 26: 5-14, 2019. PMID: 29705281. DOI: 10.1016/j.acra.2018.03.024

14 Brennan P and Silman A: Statistical methods for assessing observer variability in clinical measures. Bmj 304: 1491-1494, 1992. PMID: 1611375. DOI: 10.1136/bmj.304.6840.1491

15 Billing A, Buchner A, Stief C and Roosen A: Preoperative mp-MRI of the prostate provides little information about staging of prostate carcinoma in daily clinical practice. World J Urol 33: 923-928, 2015. PMID: 25432525. DOI: 10.1007/s00345-014-1448-5

16 Rud E, Klotz D, Rennesund K, Baco E, Johansen TEB, Diep LM, Svindland A, Eri LM and Eggesbø HB: Preoperative magnetic resonance imaging for detecting uni- and bilateral extraprostatic disease in patients with prostate cancer. World J Urol 33: 10151021, 2015. PMID: 25059766. DOI: 10.1007/ s00345-014-1362-x

17 Tan CH, Paul Hobbs B, Wei W and Kundra V: Dynamic contrastenhanced MRI for the detection of prostate cancer: MetaAnalysis. Am J Roentgenol 204: W439-W448, 2015. PMID: 25794093. DOI: $10.2214 /$ AJR.14.13373

18 Pahwa S, Schiltz NK, Ponsky LE, Lu Z, Griswold MA and Gulani V: Cost-effectiveness of MR Imaging-guided strategies for detection of prostate cancer in biopsy-naive men. Radiology 285: 157-166, 2017. PMID: 28514203. DOI: 10.1148/radiol. 2017162181

19 Tedeschi E, Palma G, Canna A, Cocozza S, Russo C, Borrelli P, Lanzillo R, Angelini V, Postiglione E, Morra VB, Salvatore M, Brunetti A and Quarantelli M: In vivo dentate nucleus MRI relaxometry correlates with previous administration of Gadolinium-based contrast agents. Eur Radiol 26: 4577-4584, 2016. PMID: 26905870. DOI: 10.1007/s00330-016-4245-2

20 McDonald RJ, McDonald JS, Kallmes DF, Jentoft ME, Murray DL, Thielen KR, Williamson EE and Eckel LJ: Intracranial gadolinium deposition after contrast-enhanced MR imaging. Radiology 275: 772-782, 2015. PMID: 25742194. DOI: 10.1148/radiol.15150025

21 Stanzione A, Cuocolo R, Cocozza S and Imbriaco M: Predicting prognosis with biparametric prostate imaging: One step at a time. Clin Genitourin Cancer, 2018. PMID: 29802061. DOI: 10.1016/j.clgc.2018.04.006

22 Stanzione A, Cocozza S, Cuocolo $\mathrm{R}$ and Imbriaco $\mathrm{M}$ : Biparametric prostate MR imaging protocol: Time to revise PIRADS Version 2? Radiology 287: 1082-1082, 2018. PMID: 29782245. DOI: $10.1148 /$ radiol.2018180292
23 Van Holsbeeck A, Degroote A, De Wever L, Vanhoutte E, De Keyzer F, Van Poppel $\mathrm{H}$ and Oyen R: Staging of prostatic carcinoma at 1.5-T MRI: correlation of a simplified MRI exam with whole-mount radical prostatectomy specimens. Br J Radiol 89: 20160101, 2016. PMID: 612500886. DOI: 10.1259/ bjr.20160101

24 Jäderling F, Nyberg T, Öberg M, Carlsson S, Skorpil M and Blomqvist L: Accuracy in local staging of prostate cancer by adding a three-dimensional $\mathrm{T} 2$-weighted sequence with radial reconstructions in magnetic resonance imaging. Acta Radiol Open 7: 205846011875460, 2018. PMID: 29456872. DOI: 10. $1177 / 2058460118754607$

25 Ruprecht O, Weisser P, Bodelle B, Ackermann H and Vogl TJ: MRI of the prostate: Interobserver agreement compared with histo-pathologic outcome after radical prostatectomy. Eur J Radiol 81: 456-460, 2012. PMID: 21354732. DOI: 10.1016/ j.ejrad.2010.12.076

26 Heijmink SWTPJ, Fütterer JJ, Hambrock T, Takahashi S, Scheenen TWJ, Huisman HJ, Hulsbergen-Van de Kaa CA, Knipscheer BC, Kiemeney LALM, Witjes JA and Barentsz JO: Prostate Cancer: Body-array versus endorectal coil MR imaging at $3 \mathrm{~T}$ - comparison of image quality, localization, and staging performance. Radiology 244: 184-195, 2007. PMID: 17495178. DOI: $10.1148 /$ radiol 2441060425

27 Jansen BHEE, Oudshoorn FHKK, Tijans AM, Yska MJ, Lont AP, Collette ERPP, Nieuwenhuijzen JA and Vis AN: Local staging with multiparametric MRI in daily clinical practice: diagnostic accuracy and evaluation of a radiologic learning curve. World J Urol 36: 1409-1415, 2018. PMID: 29680949. DOI: 10.1007/s00345-0182295-6

28 Fütterer JJ, Engelbrecht MR, Huisman HJ, Jager GJ, Hulsbergenvan De Kaa CA, Witjes JA and Barentsz JO: Staging prostate cancer with dynamic contrast-enhanced endorectal MR imaging prior to radical prostatectomy: experienced versus less experienced readers. Radiology 237: 541-549, 2005. PMID: 16244263. DOI: $10.1148 /$ radiol.2372041724

29 de Rooij M, Hamoen EHJ, Witjes JA, Barentsz JO and Rovers MM: Accuracy of magnetic resonance imaging for local staging of prostate cancer: a diagnostic meta-analysis. Eur Urol 70: 233245, 2016. PMID: 26215604. DOI: 10.1016/j.eururo.2015.07.029

30 Krishna S, Lim CS, McInnes MDF, Flood TA, Shabana WM, Lim RS and Schieda N: Evaluation of MRI for diagnosis of extraprostatic extension in prostate cancer. J Magn Reson Imaging 47: 176-185, 2018. PMID: 28387981. DOI: 10.1002/jmri.25729

31 Stanzione A, Cuocolo R, Cocozza S, Romeo V, Persico F, Fusco F, Longo N, Brunetti A and Imbriaco M: Detection of extraprostatic extension of cancer on biparametric MRI combining texture analysis and machine learning: Preliminary results. Acad Radiol pii: S1076-6332(19)30018-2, 2019. PMID: 30655050. DOI: $10.1016 /$ j.acra.2018.12.025

32 Cuocolo R, Stanzione A, Ponsiglione A, Romeo V, Verde F, Creta M, La Rocca R, Longo N, Pace L and Imbriaco M: Clinically Significant prostate cancer detection on MRI: a radiomic shape features study. Eur J Radiol 116: 144-149, 2019. PMID: 31153556. DOI: 1:0.1016/j.ejrad.2019.05.006 